Gynäkologe 2021 · 54:377

https://doi.org/10.1007/s00129-021-04823-3

Angenommen: 25. Mai 2021

Online publiziert: 1. Juni 2021

๑) Springer Medizin Verlag GmbH, ein Teil von Springer Nature 2021
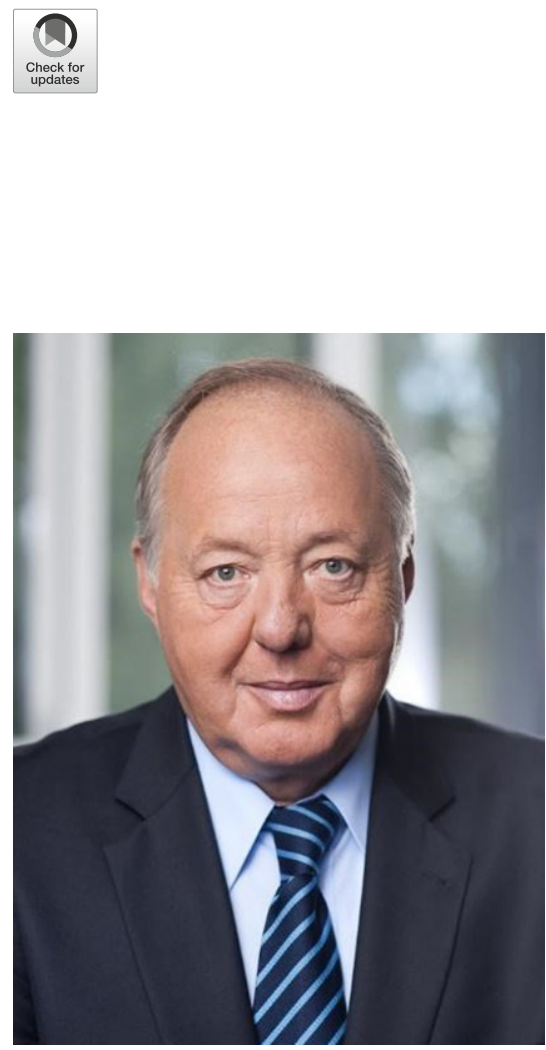

Prof. Dr. Rolf Kreienberg ist am 10. Mai 2021 in Mainz im Alter von 74 Jahren verstorben. Er war ein herausragender deutscher Gynäkologe und Onkologe und hat das hohe Ansehen der deutschen Onkologie im In- und Ausland entscheidend geprägt.

Am 27. Oktober 1946 wurde er in Mainz geboren und studierte Humanmedizin in Mainz und Wien. Nach dem Wehrdienst bei der Marine begann er 1975 als wissenschaftlicher Mitarbeiter an der Klinik für Frauenheilkunde und Geburtshilfe der Universität Mainz. Er wurde dort nach der Habilitation 1983 im Jahr 1984 zum Professor und leitenden Oberarzt unter Professor Friedberg ernannt, 1992 nahm er den Ruf auf das Direktorat der Universitätsfrauenklinik Ulm an.

In diesen Jahren baute Professor Kreienberg den Schwerpunkt seiner wissenschaftlichen Tätigkeit in der gynäkologischen Onkologie auf. Er entwi-

Klaus Diedrich ' für die Schriftleitung von Der Gynäkologe

${ }^{1}$ Klinik für Frauenheilkunde, Universitätsklinikum Schleswig-Holstein, Lübeck, Deutschland

\title{
Nachruf zum Tode von Prof. Dr. med. Rolf Kreienberg
}

\section{Oktober 1946-10. Mai 2021}

ckelte neue Operationsverfahren in der Mammachirurgie und für gynäkologische Tumoren. Ebenso verbesserte er die Chemo- und Hormontherapie beim Mammakarzinom und beschäftigte sich mit der Immunologie gynäkologischer Tumoren und der Bedeutung von Tumormarkern. In diesen Bereichen gestaltete er auch federführend einige Themenhefte von Der Gynäkologe. Seinen ersten Artikel in der Zeitschrift veröffentlichte er 1979: „Intrauteriner Abort und Extrauteringravidität mit tödlichem Ausgang“. Er trat im Jahr 2002 als Herausgeber ins Board der Zeitschrift ein und betreute insgesamt 32 Schwerpunkthefte federführend oder als Ko-Herausgeber. Sein erstes Heft zum Thema Karzinomnachsorge betreute er 1989 gemeinsam mit Prof. Volker Friedberg. Im Board engagierte er sich nicht nur als Herausgeber, sondern auch in den Rubriken „CME Zertifizierte Fortbildung“, „Bild und Fall“ sowie „Handlungsalgorithmus".

Als Anerkennung für die großartige wissenschaftliche Leistung in der Gynäkologie wurde ihm 2008 die Präsidentschaft der Deutschen Gesellschaft für Gynäkologie und Geburtshilfe (DGGG) angetragen. Vorher war er von 2000-2002 Präsident der Deutschen Krebsgesellschaft (DKG). In letzterer hatte er sich aktiv für den Aufbau des Zertifizierungssystems eingesetzt, wofür er 2018 mit der Karl-Heinrich-Bauer-Medaille der DKG ausgezeichnet wurde.

In der wissenschaftlichen Gesellschaft hat Professor Kreienberg entscheidend die Entstehung der wichtigen Leitlinien weiterentwickelt und wurde deshalb 2012 auch Vorsitzender der Leitlinien- kommission sowie 2015 Präsident der Arbeitsgemeinschaft der Wissenschaftlichen Medizinischen Fachgesellschaften (AWMF).

Neben seiner hervorragenden Tätigkeit in der gynäkologischen Onkologie war es immer eine große Freude und Bereicherung, im Freundeskreis privat mit ihm zusammen zu sein. Er war ein guter Freund, empathischer Arzt, engagierter Forscher und akademischer Lehrer. Unser Mitgefühl gehört seiner Frau Dagmar und seiner Familie. Die deutsche Frauenheilkunde verliert mit Professor Kreienberg einen allseits geschätzten Arzt und Forscher. Professor Kreienberg wird uns als Vorbild fehlen und wird uns als guter Freund stets in Erinnerung bleiben.

Im Namen aller Herausgeber*innen der Zeitschrift Der Gynäkologe, dem Fortbildungsorgan der Deutschen Gesellschaft für Gynäkologie und Geburtshilfe,

Editor-in-Chief

Prof. Dr. med. Dr. h.c. mult. Klaus Diedrich

\section{Korrespondenzadresse}

Prof. Dr. med. Dr. h.c. mult. Klaus Diedrich Klinik für Frauenheilkunde, Universitätsklinikum Schleswig-Holstein

Ratzeburger Allee 160, 23538 Lübeck,

Deutschland

prof.diedrich@gmail.com 